\title{
ACYCLIC COLOURINGS OF PLANAR GRAPHS WITH LARGE GIRTH
}

\author{
O. V. BORODIN, A. V. KOSTOCHKA AND D. R. WOODALL
}

\begin{abstract}
A proper vertex-colouring of a graph is acyclic if there are no 2-coloured cycles. It is known that every planar graph is acyclically 5-colourable, and that there are planar graphs with acyclic chromatic number $\chi_{\mathrm{a}}=5$ and girth $g=4$. It is proved here that a planar graph satisfies $\chi_{\mathrm{a}} \leqslant 4$ if $g \geqslant 5$ and $\chi_{\mathrm{a}} \leqslant 3$ if $g \geqslant 7$.
\end{abstract}

\section{Introduction}

An acyclic colouring of a graph $G$ is a proper vertex-colouring of $G$ such that every union of two colour classes induces an acyclic subgraph of $G$, and $\chi_{\mathrm{a}}=\chi_{\mathrm{a}}(G)$ denotes the smallest number of colours in an acyclic colouring of $G$. Clearly $\chi_{\mathrm{a}}(C)=3$ if $C$ is a cycle and $\chi_{\mathrm{a}}(F) \leqslant 2$ if $F$ is a forest, with equality unless $F$ is edgeless.

For a planar graph $G$, Grünbaum [5] conjectured that $\chi_{\mathrm{a}}(G) \leqslant 5$ and proved that $\chi_{\mathrm{a}}(G) \leqslant 9$. This bound was sharpened by Mitchem [9] to 8, by Albertson and Berman [1] to 7, by Kostochka [7] to 6 , and by Borodin [3, 4] to 5, which is best possible since the double 5 -wheel $C_{5}+\bar{K}_{2}$ is planar and (it is easy to see) has $\chi_{\mathrm{a}}=5$.

The girth $g=g(G)$ of a graph $G$ is the length of its shortest cycle. The purpose of the present paper is to prove the following two results, which were partly inspired by J. Nešetřil telling us of Fact 4 (below).

THEOREM 1. If $G$ is planar with girth $g \geqslant 5$ then $\chi_{\mathrm{a}} \leqslant 4$.

THEOREM 2. If $G$ is planar with girth $g \geqslant 7$ then $\chi_{\mathrm{a}} \leqslant 3$.

Kostochka and Melnikov [8] have constructed planar 2-degenerate bipartite graphs, necessarily with girth $g=4$, having $\chi_{\mathrm{a}}=5$. (For example, in $C_{5}+\bar{K}_{2}$, replace each edge $u v$ of $C_{5}$ by a copy of $K_{2,4}$ with $u, v$ as the vertices of degree 4.) Thus our condition $g \geqslant 5$ is best possible to imply $\chi_{\mathrm{a}} \leqslant 4$. However, we do not know whether $\chi_{\mathrm{a}} \leqslant 3$ whenever $g \geqslant 6$ (or even $g \geqslant 5$ ).

Theorems 1 and 2 have several corollaries, in view of the following facts.

Received 20 December 1995; revised 26 October 1997.

1991 Mathematics Subject Classification 05C15.

This work was carried out while the first author was visiting Nottingham, funded by Visiting Fellowship Research Grant GR/K00561 from the Engineering and Physical Sciences Research Council. The work of this author was also partly supported by grant NQ4300 of the International Science Foundation and the Russian Government. The work of the second author was partly supported by grant 93-01-01486 of the Russian Foundation of Fundamental Research and grant RPY300 of the International Science Foundation and the Russian Government.

J. London Math. Soc. (2) 60 (1999) 344-352 
FACT 1 (obvious). If $\chi_{\mathrm{a}}(G) \leqslant k$ then $G$ contains an induced forest on at least $2 / k$ of its vertices.

FACT 2 (S. L. Hakimi, J. Mitchem and E. S. Schmeichel (see [6])). If $\chi_{\mathrm{a}}(G) \leqslant k$ then $E(G)$ can be partitioned into $k$ 'star forests' (forests in which each component is a star).

FACT 3 (Grünbaum [5]). If $\chi_{\mathrm{a}}(G) \leqslant k$ then the star chromatic number $\chi_{\mathrm{s}}(G) \leqslant$ $k \cdot 2^{k-1}$.

FACT 4 (Raspaud and Sopena [10]). If $\chi_{\mathrm{a}}(G) \leqslant k$ then the oriented chromatic number $\chi_{0}(G) \leqslant k \cdot 2^{k-1}$.

By Fact 2, Borodin's 5-colour theorem implies the truth of the conjecture of Algor and Alon [2] that the edges of every planar graph can be partitioned into five star forests. By Facts 3 and 4 , it also implies that $\chi_{\mathrm{s}}(G) \leqslant 80$ and $\chi_{\mathrm{o}}(G) \leqslant 80$ for every planar graph $G$; these bounds remain the best known. For girth $g \geqslant 5$, Theorem 1 gives $\chi_{\mathrm{s}}(G) \leqslant 32$ and $\chi_{\mathrm{o}}(G) \leqslant 32$; for $g \geqslant 7$, Theorem 2 gives $\chi_{\mathrm{s}}(G) \leqslant 12$ and $\chi_{\mathrm{o}}(G) \leqslant$ 12.

\section{Preliminaries}

The proofs of the two theorems have a similar structure. In each case we let $G$ be a smallest counterexample to the theorem, which we assume is already embedded in the plane, and we note that clearly $G$ is 2-connected. Our proof uses an application of Euler's formula (Lemma 1) and some structural information derived from the minimality of $G$ (Lemmas 2-5); we then use the method of redistribution of charge in order to obtain a contradiction.

Throughout, $G$ has $n$ vertices, $m$ edges and $r$ faces, the sets of which are denoted by $V, E$ and $F$ respectively. The degree of vertex $v$ is denoted by $d(v)$, a $d$-vertex is a vertex $v$ with $d(v)=d$, and a $d(b)$-vertex is a $d$-vertex that is adjacent to exactly $b$ vertices of degree 2. The number of edges incident to face $f$ is denoted by $r(f)$, and an $r$-face or $>r$-face is a face $f$ with $r(f)=r$ or $r(f)>r$, respectively. An (alternating) $i, j$-path is a path whose vertices are coloured alternately $i$ and $j$. A cycle $C$ separates two vertices if one of the vertices is inside $C$ and the other is outside $C$, and a separating cycle is a cycle that separates some two vertices. The following lemma holds for every connected planar graph.

LEMMA 1.

(i) $\sum_{v \in V}(3 d(v)-10)+\sum_{f \in F}(2 r(f)-10)=-20$.

(ii) $\sum_{v \in V}(5 d(v)-14)+\sum_{f \in F}(2 r(f)-14)=-28$.

Proof. Euler's formula $n-m+r=2$ can be rewritten in the form $(6 m-10 n)+(4 m-10 r)=-20$, which implies (i), and in the form $(10 m-14 n)+(4 m-14 r)=-28$, which implies (ii).

\section{Proof of Theorem $1(g \geqslant 5)$}

Let $G$ be a smallest counterexample to Theorem 1. As noted above, $G$ is 2connected and so has minimum degree at least 2 . 
Lemma 2. (i) No 2-vertex is adjacent to a 2-vertex or 3-vertex.

(ii) G contains no $d(d)$-vertices $(2 \leqslant d \leqslant 15)$, no $d(d-1)$-vertices $(2 \leqslant d \leqslant 9)$ and no $d(d-2)$-vertices $(3 \leqslant d \leqslant 4)$.

(iii) If $w$ is a 5(3)-vertex, then the three 2-vertices occur consecutively in cyclic order round $w$, and both of the two faces between consecutive 2-vertices are $>5$-faces.

(iv) If a 5(2)-vertex is adjacent to three 3-vertices, then it is incident to at least one $>$ 5-face.

(v) A 5(3) or 6(4)-vertex is not adjacent to any 3-vertices.

Proof. (i): (i) follows immediately from (ii). In proving (ii)-(v), we assume throughout that $w$ is a $d(b)$-vertex with neighbours $v_{1}, \ldots, v_{b}, z_{1}, \ldots, z_{d-b}$ where $v_{1}, \ldots$, $v_{b}$ have degree 2 and are adjacent to $u_{1}, \ldots, u_{b}$ respectively. The neighbours of $z_{i}$ other than $w$ will be referred to as the outer neighbours of $z_{i}(1 \leqslant i \leqslant d-b)$. By the minimality of $G$, we may suppose that $G-v_{1}$ has an acyclic 4-colouring $c: \bigvee\left\{v_{1}\right\} \longrightarrow$ $\{1,2,3,4\}$ in which without loss of generality $c(w)=1$. If we can convert this into an acyclic 4-colouring of $G$ by colouring $v_{1}$ (perhaps after first recolouring some other vertices), then this contradiction will complete the proof. Note that if $c\left(u_{i}\right) \neq c(w)$ then we can give $v_{i}$ either of the other colours since no 2-coloured cycle can possibly use $v_{i}$. Thus we may suppose that $c\left(u_{1}\right)=1$, and that for $j=2,3,4$ there is an alternating $1, j$-path connecting $u_{1}$ to $w$ (since otherwise we could set $c\left(v_{1}\right)=j$ ).

(ii) and (iii): If $b=d<4^{2}$, then choose a colour $j$ that appears on at most three of $u_{1}, \ldots, u_{b}$. Set $c(w)=j$, give the intervening $v_{i}$ distinct colours not equal to $j$, and give the remaining $v_{i}$ any proper colours; this colouring is clearly acyclic.

If $b=d-1<3^{2}$, then choose a colour $j \neq c\left(z_{1}\right)$ that appears on at most two of $u_{1}, \ldots, u_{b}$. Set $c(w)=j$, and proceed as before. If $b=d-2<2^{2}$, then the same trick works provided that $c\left(z_{1}\right) \neq c\left(z_{2}\right)$, but if $c\left(z_{1}\right)=c\left(z_{2}\right)$ then we dare not recolour $w$ for fear of creating a 2-coloured cycle. However, if at most two of $u_{1}, \ldots, u_{b}$ have colour 1 , which must be the case if $d-2 \leqslant 2$, then we can colour the corresponding $v_{i}$ with distinct colours not in $\left\{1, c\left(z_{1}\right)\right\}$. This proves (ii), and it also shows that in proving (iii) we may assume that $c\left(z_{1}\right)=c\left(z_{2}\right)=2$, say, and that $c\left(u_{i}\right)=1$ for all $i$. Hence if $v_{i}, v_{j}$ occur consecutively in cyclic order round $w$, then there is a $>5$-face between them (otherwise $u_{i} u_{j} \in E$ ).

If the $v_{i}$ are not consecutive in cyclic order round $w$, assume that $v_{1}$ is between $z_{1}$ and $z_{2}$. Because of the 1,4-path connecting $u_{1}$ to $w$, there can be no 2,3-path from $z_{1}$ to $z_{2}$. Thus we may give $w$ colour 3 and the $v_{i}$ any proper colours. This proves (iii).

(iv): Suppose that $(d, b)=(5,2), d\left(z_{i}\right)=3(i=1,2,3)$ and $w$ is incident to five 5faces. If $c\left(u_{2}\right)=1$ then, because of the 5 -faces, $v_{1}$ and $v_{2}$ are not consecutive in cyclic order round $w$, and at most one of $z_{1}, z_{2}, z_{3}$ has an outer neighbour coloured 1, but this contradicts the existence of the three $1, j$-paths connecting $u_{1}$ to $w$, so we may suppose that $c\left(u_{2}\right) \neq 1$. Then without loss of generality $c\left(z_{i}\right)=i+1$ and $z_{i}$ has an outer neighbour coloured 1 (because of the $1,(i+1)$-path, $i=1,2,3)$. Choose a colour $j \notin\left\{1, c\left(u_{2}\right)\right\}$ that occurs on at most one of the outer neighbours of $z_{1}, z_{2}$ and $z_{3}$, set $c(w)=j$ and give $z_{j-1}, v_{1}$ and $v_{2}$ any proper colours.

(v): Suppose that $(d, b)=(5,3)$ or $(6,4)$ and $d\left(z_{1}\right)=3$. First suppose that $c\left(z_{1}\right)=$ $c\left(z_{2}\right)$. If the two outer neighbours of $z_{1}$ have the same colour $j$, we may choose $c(w) \notin\left\{j, c\left(z_{1}\right)\right\}$ such that $c(w)$ occurs on at most two of $u_{1}, \ldots, u_{b}$; the $v_{i}$ are now easily coloured. If the two outer neighbours of $z_{1}$ have distinct colours, we may recolour first $z_{1}$ and then $w$, and so we may assume from now on that $c\left(z_{1}\right) \neq c\left(z_{2}\right)$, without loss of generality $c\left(z_{i}\right)=i+2(i=1,2)$. If $c\left(u_{i}\right)=1$ for at most one $i$, put $c(w)=1$ and 
$c\left(v_{i}\right)=2$. The same works with 1 and 2 interchanged, and so we may suppose that $(d, b)=(6,4), c\left(u_{1}\right)=c\left(u_{2}\right)=1$ and $c\left(u_{3}\right)=c\left(u_{4}\right)=2$. If $z_{1}$ has no outer neighbour coloured 1 , we may put $c(w)=1, c\left(v_{1}\right)=2, c\left(v_{2}\right)=3$. The same again works with 1 and 2 interchanged, and so we may suppose that $z_{1}$ has outer neighbours coloured 1 and 2. Now put $c\left(z_{1}\right)=4, c(w)=3$ and give $v_{1}, \ldots, v_{4}$ any proper colours.

By a weak vertex we mean a vertex of degree 2 or 3 or a 4 -vertex that is adjacent to both a 2 -vertex and a 3-vertex.

Lemma 3. Each 3-vertex is adjacent to at most one weak vertex

Proof. Let $w$ be a 3-vertex adjacent to $x, y, z$ where $x, y$ are weak, with degree 3 or 4 (by Lemma 2(i)). Let the outer neighbours of $x$ (that is, its neighbours other than w) be $x_{1}, x_{2}$ and, if $d(x)=4, x_{3}$, where $d\left(x_{3}\right)=2$ and the other neighbour of $x_{3}$ is $x_{3}^{\prime}$. To avoid referring to non-existent vertices, if $d(x)=3$ add isolated vertices $x_{3}, x_{3}^{\prime}$ to $G$. Deal with $y$ analogously. Let $c$ be an acyclic 4-colouring of $G-\left\{w, x_{3}, y_{3}\right\}$. In what follows, whenever we describe how to colour $x_{3}$, we assume implicitly that $c\left(x_{3}^{\prime}\right)=$ $c(x)$, since if $c\left(x_{3}^{\prime}\right) \neq c(x)$ then we can use either of the other colours for $c\left(x_{3}\right)$ with impunity; similarly with $y_{3}$. Assume that $c(z)=1$. By interchanging $x, y$ and permuting the other colours if necessary, we have only four cases to consider.

Case 1: $c(x)=2, c(y)=3$. Set $c(w)=4$, choose $c\left(x_{3}\right) \notin\left\{c(x), c\left(x_{1}\right), c\left(x_{2}\right)\right\}$, and colour $y_{3}$ similarly.

Case 2: $c(x)=c(y)=2$. If $c\left(x_{1}\right) \neq c\left(x_{2}\right)$ and $\left\{c\left(x_{1}\right), c\left(x_{2}\right)\right\} \neq\{3,4\}$, then change $c(x)$ to get case 1 . Hence we may assume that $c\left(x_{1}\right)=c\left(x_{2}\right)$ or $\left\{c\left(x_{1}\right), c\left(x_{2}\right)\right\}=\{3,4\}$, and similarly for $y_{1}, y_{2}$. If there is no 2,3-path connecting $x$ to $y$, set $c(w)=3$, if $c\left(x_{1}\right)=$ $c\left(x_{2}\right)$ choose $c\left(x_{3}\right) \notin\left\{c(x), c\left(x_{1}\right), c(w)\right\}$, if $\left\{c\left(x_{1}\right), c\left(x_{2}\right)\right\}=\{3,4\}$ set $c\left(x_{3}\right)=1$, and colour $y_{3}$ similarly. We can do the same if there is no 2,4-path connecting $x$ to $y$; hence we may suppose that both paths exist and $c\left(x_{1}\right)=c\left(y_{1}\right)=3, c\left(x_{2}\right)=c\left(y_{2}\right)=4$. Now, either the 2,3-path (completed to a cycle through $w$ ) separates $x_{2}$ from $z$ or the 2,4path (similarly completed) separates $x_{1}$ from $z$. Suppose the former, so that there is no 1,4-path connecting $x_{2}$ to $z$; set $c(w)=4, c(x)=1, c\left(x_{3}\right)=2$ and $c\left(y_{3}\right)=1$.

Case 3: $c(x)=1, c(y)=2$. If $c\left(x_{1}\right) \neq c\left(x_{2}\right)$ we can change $c(x)$ to get case 1 or case 2. Hence assume that $c\left(x_{1}\right)=c\left(x_{2}\right) \neq 3$ and choose $c(w)=3, c\left(x_{3}\right) \notin\{c(w), c(x)$, $\left.c\left(x_{1}\right)\right\}, c\left(y_{3}\right) \notin\left\{c(y), c\left(y_{1}\right), c\left(y_{2}\right)\right\}$.

Case 4: $c(x)=c(y)=1$. As in case 3, we may suppose that $c\left(x_{1}\right)=c\left(x_{2}\right)$, and similarly $c\left(y_{1}\right)=c\left(y_{2}\right)$. Choose $c(w) \notin\left(1, c\left(x_{1}\right), c\left(y_{1}\right)\right\}, c\left(x_{3}\right) \notin\left\{c(w), c(x), c\left(x_{1}\right)\right\}$ and $c\left(y_{3}\right) \notin\left\{c(w), c(y), c\left(y_{1}\right)\right\}$.

We now show that Lemmas 2 and 3 contradict the supposition that $g \geqslant 5$. Assign a 'charge' of $3 d(v)-10$ units to each vertex $v$ of $G$ and of $2 r(f)-10$ units to each face $f$ of $G$. By Lemma 1(i), the total charge assigned is negative. We now redistribute the charge, without changing its sum, in such a way that the sum is provably nonnegative, and this contradiction will prove the theorem. Note that the charge on each face is non-negative, by the supposition that $r(f) \geqslant g \geqslant 5$, and vertices of degree 2 , $3,4,5, \ldots$ start with charge $-4,-1,2,5, \ldots$. 
The rules for redistribution are as follows:

(R1) Each 2-vertex receives 2 from each adjacent vertex.

(R2) Each 3 -vertex receives $\frac{1}{2}$ from each adjacent non-weak vertex.

(R3) Each face $f$ with $r(f)>5$ and bounding cycle $v_{1} v_{2} \ldots v_{r(f)} v_{1}$ gives $\frac{1}{2}$ to each vertex $v_{i}$ for which $d\left(v_{i-1}\right) \leqslant 3$ and $d\left(v_{i+1}\right) \leqslant 3$ (subscripts modulo $r(f)$ ).

It is easy to see that the charge on each face $f$ is still non-negative: by Lemmas 2(i) and 3 , the boundary of $f$ cannot contain three consecutive vertices with degree $\leqslant 3$, and so $f$ cannot contribute $\frac{1}{2}$ to two adjacent vertices in its boundary; thus $f$ gives up at most $\frac{1}{4} r(f)$, whereas its initial charge was $2 r(f)-10>\frac{1}{4} r(f)$ if $r(f)>5$.

It remains to prove that the charge on each vertex $v$ is also non-negative. If $d(v)=2$ then $v$ started with charge -4 and has gained 4 , and so now has charge 0 . If $d(v)=3$ then $v$ started with -1 and has gained at least 1 by Lemma 3 , and so it now has non-negative charge. Suppose that $d(v)=4$, so that $v$ started with charge 2 . By Lemma 2(ii) and the definition of a weak vertex, if $v$ is adjacent to a 2-vertex then it gave 2 to only one 2-vertex and nothing to 3-vertices; otherwise it gave $\frac{1}{2}$ to at most four 3-vertices. In either case its charge is still non-negative.

Suppose that $d(v)=5$, so that $v$ is a $5(b)$-vertex where $b \leqslant 3$ by Lemma 2(ii). If $b=3$ then, by Lemma 2(iii) and (v), $v$ received $\frac{1}{2}$ from two $>5$-faces, between pairs of 2-vertices, and gave nothing to 3-vertices; thus $v$ started with charge 5, gave 6 to three 2-vertices, received 1 from faces, and now has 0 . If $b=2$ then $v$ gave 4 to 2vertices and, by Lemma 2(iv), it either gave at most 1 to 3 -vertices or gave $1 \frac{1}{2}$ to 3 vertices and received $\frac{1}{2}$ from a $>5$-face. If $b \leqslant 1$ then $v$ gave at most 2 to a 2 -vertex plus 2 to four 3 -vertices.

If $d(v)=6$ then $v$ started with 8 and, by Lemma 2(ii) and (v), gave at most 8 , either to four 2-vertices, or to at most three 2 -vertices and three 3 -vertices. If $7 \leqslant d(v) \leqslant 9$ then, by Lemma 2(ii), $v$ gave to at most $d(v)-2$ 2-vertices and two 3 -vertices, making a total of at most $2 d(v)-3 \leqslant 3 d(v)-10$. Finally, if $d(v) \geqslant 10$ then $v$ gave at most $2 d(v)$ $\leqslant 3 d(v)-10$. Thus every vertex now has non-negative charge, and this contradiction completes the proof of Theorem 1 .

\section{Proof of Theorem $2(g \geqslant 7)$}

Let $G$ be a smallest counterexample to Theorem $2 ; G$ is 2-connected, with minimum degree at least 2 .

Lemma 4. (i) $G$ does not contain two adjacent 2-vertices.

(ii) $G$ contains no $d(d)$-vertices $(2 \leqslant d \leqslant 8)$ or $d(d-1)$-vertices $(2 \leqslant d \leqslant 4)$.

(iii) No 3-vertex is adjacent to three 3(1)-vertices.

(iv) No 3(1)-vertex is adjacent to two 3(1)-vertices.

Proof. (i) and (ii): With the terminology of Lemma 2, if $b=d<3^{2}$ then choose a colour $j$ that occurs on at most two of $u_{1}, \ldots, u_{b}$. If $b=d-1<2^{2}$ then choose a colour $j \neq c\left(z_{1}\right)$ that occurs on at most one of $u_{1}, \ldots, u_{b}$. In each case, set $c(w)=j$ and proceed as in Lemma 2(ii). This proves (ii), and (i) immediately follows.

(iii): For $i=1,2,3$, let $G$ contain paths $w x_{i} v_{i} u_{i}$ where $d(w)=3, d\left(v_{i}\right)=2, x_{i}$ has another neighbour $y_{i}$, and distinct labels denote distinct vertices. Let $c$ be an acyclic 3 -colouring of $G-\left\{w, v_{1}, v_{2}, v_{3}, x_{1}, x_{2}, x_{3}\right\}$. 
Suppose that we colour $w$. If $c(w) \neq c\left(y_{i}\right)$, say $c(w)=1$ and $c\left(y_{i}\right)=2$, we can colour the path $w x_{i} v_{i} u_{i}$ either 1321 or 1312 or 1313 depending on the colour of $u_{i}$, and only in the last case is there an alternating path through $x_{i}$; this is a $c(w), c\left(u_{i}\right)$-path and requires $w, y_{i}$ and $u_{i}$ to have three different colours. If $c(w)=c\left(y_{i}\right)$ then, by choosing $c\left(v_{i}\right) \neq c(w)$ if $c\left(x_{i}\right)=c\left(u_{i}\right)$, we can ensure that there is only the inevitable $c(w), c\left(x_{i}\right)$-path through $c\left(x_{i}\right)$; this works for either of the two possible choices for $c\left(x_{i}\right)$.

We now colour $w$ as follows; in each case, by the above remarks, we can colour the $x_{i}$ and $v_{i}$ so as to create no 2-coloured cycles. If $c\left(y_{i}\right)=1$, say, for each $i$, let $c(w)$ be whichever of 2,3 occurs on more of the $u_{i}$ (so that the other occurs on at most one $\left.u_{i}\right)$. If $c\left(y_{1}\right)=c\left(y_{2}\right)=1$ and $c\left(y_{3}\right)=2$, set $c(w)=3$ unless $c\left(u_{1}\right)=c\left(u_{2}\right)=2$, in which case set $c(w)=2$. If $c\left(y_{i}\right)=i$ for each $i$, set $c(w)=j$ where $j$ is chosen so that $\left\{j, c\left(y_{i}\right), c\left(u_{i}\right)\right\}=\{1,2,3\}$ for at most one $i$, and choose $c\left(x_{j}\right) \neq c\left(u_{i}\right)$ if there is such an $i$.

(iv): This is essentially the same as (iii) with $u_{3}, v_{3}$ removed and $c\left(u_{3}\right)$ interpreted as 1 , say, whenever it occurs in the above argument.

Recall that $G$ has girth $g(G)=g \geqslant 7$. An $r$-cycle, $\leqslant r$-cycle or $<r$-cycle is a cycle with length $l=r, l \leqslant r$ or $l<r$, respectively. A $*$-cycle is a separating $r$-cycle, where $r=7$ or 8 . If $G$ contains a *-cycle, then let $S$ be a ${ }^{*}$-cycle with as few vertices as possible inside it, and describe every vertex inside $S$ as distinguished; otherwise, every vertex of $G$ is distinguished.

Lemma 5. (i) If $a^{*}$-cycle $C$ passes through a distinguished vertex, then $C$ is an 8cycle.

(ii) If two distinguished 3(1)-vertices $b_{1}, b_{2}$ are adjacent then edge $b_{1} b_{2}$ is incident with $a>7$-face.

Proof. (i): If such a $C$ exists then clearly $S$ exists and $C \cap S \neq \varnothing$. Suppose that $C$ is a 7-cycle. If only one vertex of $C$ is inside or outside $S$, then combined with a segment of $S$ it gives a $\leqslant 6$-cycle, contradicting $g \geqslant 7$. Thus either two or three vertices of $C$ are inside $S,|V(S)|=8$, and $C$ splits $S$ into two equal segments, creating two 7-cycles or 8-cycles with fewer vertices inside them than $S$. Clearly these cycles can have no chords, and since no two 2-vertices of $G$ are adjacent by Lemma 4(i), at least one of the cycles must be separating, contradicting the definition of $S$.

(ii): For $i=1,2$, let $b_{i}$ be adjacent to $h_{i}$ and $k_{i}$ where $d\left(k_{i}\right)=2$. There are two cases.

Case 1: $k_{1}, k_{2}$ are incident with the same face. Assume that this is labelled as in Figure 1(a). Form $G_{i}$ from $G^{\prime}=G-\left\{k_{1}, b_{1}, b_{2}, k_{2}\right\}$ by adding a new 2-vertex $z_{i}$ adjacent to $f_{i}$ and $h_{3-i}(i=1,2)$.

CLAIM 1. Either $g\left(G_{1}\right) \geqslant 7$ or $g\left(G_{2}\right) \geqslant 7$.

Proof. Suppose that $g\left(G_{1}\right) \leqslant 6$ and $g\left(G_{2}\right) \leqslant 6$. Then $G^{\prime}$ contains paths $f_{1} u_{1} \ldots h_{2}$ and $f_{2} u_{2} \ldots h_{1}$ of length at most 4 . These paths must cross, at a vertex $v$, say. The distances from $v$ along these paths satisfy $d\left(v, f_{1}\right)+d\left(v, h_{2}\right) \leqslant 4$ and $d\left(v, f_{2}\right)+d\left(v, h_{1}\right) \leqslant 4$ by assumption, and also $d\left(v, f_{1}\right)+d\left(v, h_{1}\right) \geqslant 4, d\left(v, f_{2}\right)+d\left(v, h_{2}\right) \geqslant 4$ and $d\left(v, h_{1}\right)+d\left(v, h_{2}\right) \geqslant$ 4 because $g(G) \geqslant 7$. It follows that either $d\left(v, f_{1}\right)=d\left(v, f_{2}\right)=1$ and $d\left(v, h_{1}\right)=d\left(v, h_{2}\right)=$ 3 , or else all four distances equal 2. In the first case, $v=u_{1}=u_{2}$ and we have a 4-cycle 


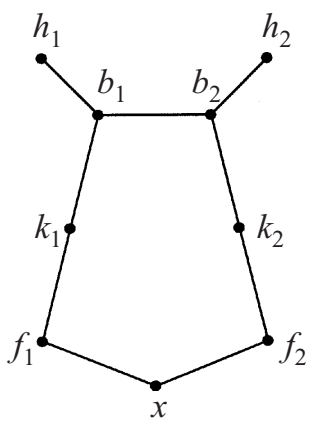

(a)

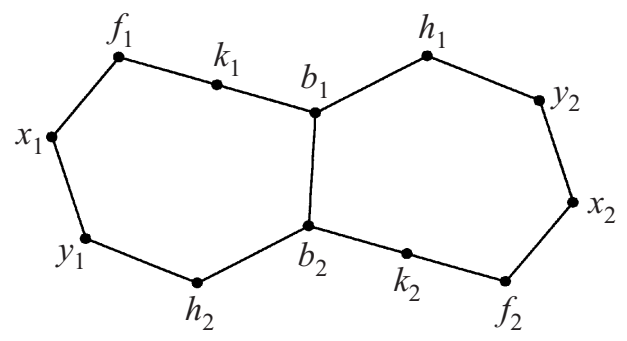

(b)

FIGURE 1.

unless $v=x$. In the second, $x f_{1} u_{1} v u_{2} f_{2} x$ is a closed walk of length 6 , which contains a $\leqslant 6$-cycle unless $u_{1}=u_{2}=x$. In either case we may suppose that $u_{1}=x$. Then there is a 7-cycle $h_{2} b_{2} k_{2} f_{2} x \ldots h_{2}$, which is separating because $d\left(f_{2}\right) \neq d\left(k_{2}\right)=2$ by Lemma 4(i). This contradicts Lemma 5(i), and so completes the proof of the claim.

By Claim 1, we may suppose without loss of generality that $g\left(G_{1}\right) \geqslant 7$, which means that $G_{1}$ has an acyclic 3-colouring $c$ by the minimality of $G$. We now show that this can be modified into an acyclic 3-colouring of $G$. As in Lemma 3, whenever we describe how to colour $k_{i}$, we assume implicitly that $c\left(b_{i}\right)=c\left(f_{i}\right)$, since otherwise $c\left(k_{i}\right)$ is uniquely determined and no 2-coloured cycle can possibly use $k_{i}$.

Without loss of generality $c\left(f_{1}\right)=1$. If $c\left(h_{2}\right) \neq 1$, say $c\left(h_{2}\right)=2$, we can colour $b_{1} b_{2} k_{2}$ so that $h_{1} b_{1} b_{2} k_{2}$ is coloured 1231,2313 or 3213 , depending on $c\left(h_{1}\right)$. Thus we may suppose that $c\left(h_{2}\right)=c\left(f_{1}\right)=1$ and, by symmetry, that $c\left(h_{1}\right)=c\left(f_{2}\right)=j$, say. If $j=1$, set $c\left(b_{1}\right)=2, c\left(b_{2}\right)=3$. Suppose $j \neq 1$, say $j=2$. If in $G_{1}, c\left(z_{1}\right)=3$, colour $k_{1} b_{1} b_{2}$ with 313. Otherwise, $c\left(z_{1}\right)=2$, and we colour $k_{1} b_{1} b_{2}$ with 213 or 312 according to whether there is or is not a 1,2-path connecting $h_{1}$ to $h_{2}$; note that if there is, then there is no 1, 2-path connecting $h_{1}$ to $f_{1}$, since there is none in $G_{1}-z_{1}$ connecting $f_{1}$ to $h_{2}$. Thus in every case we have constructed an acyclic 3-colouring of $G$, and this contradiction completes the discussion of case (1).

Case 2: $k_{1}, k_{2}$ are not incident with the same face. Assume that the two faces incident to $b_{1} b_{2}$ are labelled as in Figure 1(b).

Let $c$ be an acyclic 3-colouring of $G^{\prime}=G-\left\{k_{1}, b_{1}, b_{2}, k_{2}\right\}$. If $c\left(f_{1}\right) \neq c\left(h_{2}\right)$, say $c\left(f_{1}\right)=1, c\left(h_{2}\right)=2$, then we can colour $b_{1} b_{2} k_{2}$ so that $h_{1} b_{1} b_{2} k_{2}$ is coloured 1231, 2313 or 3213 , depending on $c\left(h_{1}\right)$ (with the usual convention about colouring 2 -vertices). Thus we may suppose that in every colouring of $G^{\prime}, c\left(f_{1}\right)=c\left(h_{2}\right)$. This means that identifying $f_{1}$ with $y_{1}$ in $G^{\prime}$ must create a $\leqslant 6$-cycle, and likewise identifying $x_{1}$ with $h_{2}$.

Therefore $G^{\prime}$ contains paths $P_{1}, P_{2}$ of length at most 6 connecting $f_{1}$ to $y_{1}$ and $x_{1}$ to $h_{2}$, and $P_{1}$ and $P_{2}$ must cross, at a vertex $v$, say. The distances from $v$ along these paths satisfy $d\left(v, f_{1}\right)+d\left(v, y_{1}\right) \leqslant 6$ and $d\left(v, x_{1}\right)+d\left(v, h_{2}\right) \leqslant 6$, and also $d\left(v, f_{1}\right)+d\left(v, x_{1}\right) \geqslant$ $6, d\left(v, x_{1}\right)+d\left(v, y_{1}\right) \geqslant 6, d\left(v, y_{1}\right)+d\left(v, h_{2}\right) \geqslant 6$ and $d\left(v, f_{1}\right)+d\left(v, h_{2}\right) \geqslant 4$ because $g(G) \geqslant$ 7. It follows that either all four distances equal 3 , or else $d\left(v, f_{1}\right)=d\left(v, h_{2}\right)=2$ and 
$d\left(v, x_{1}\right)=d\left(v, y_{1}\right)=4$. Let $C_{1}, C_{2}$ and $C_{3}$ be the three cycles generated by adding $f_{1} x_{1}, x_{1} y_{1}$ and $y_{1} h_{2}$, respectively, to $P_{1} \cup P_{2}$, and let $C_{4}$ be their mod-2-sum, which is a cycle including $v$ and the path $f_{1} x_{1} y_{1} h_{2}$. The lengths of $C_{1}, \ldots, C_{4}$ are either 7,7,7,9 or 7,9,7,7; hence these cycles have no chords. $C_{4}$ is certainly separating. Since no two 2-vertices of $G$ are adjacent by Lemma 4(i), either $C_{1}$ and $C_{3}$ are both separating or $C_{2}$ is separating. Either way, each of $x_{1}$ and $y_{1}$ lies on a separating 7-cycle, and so $S$ exists and, by Lemma 5(i), neither of these vertices is inside $S$. However, $b_{1}$ and $b_{2}$ are inside $S$, and so all vertices in Figure 1(b) are inside $S$ or on $S$. Hence $x_{1}$ and $y_{1}$ are on $S$.

Similarly, $x_{2}$ and $y_{2}$ are on $S$. Thus $S$ contains at least two internally disjoint paths between $\left\{x_{1}, y_{1}\right\}$ and $\left\{x_{2}, y_{2}\right\}$, at least one of which, say $P$, has at most two internal vertices. Without loss of generality $P$ connects $x_{1}$ to $y_{2}$. Then we have a $\leqslant 8$-cycle $x_{1} f_{1} k_{1} b_{1} h_{1} y_{2} P x_{1}$ which is strictly enclosed in $S$, and is separating because $d\left(f_{1}\right) \neq$ $d\left(k_{1}\right)=2$ by Lemma 4(i). This contradicts the definition of $S$ and so completes the proof of Lemma 5.

We now show that Lemmas 4 and 5 give a contradiction. If $G$ contains a *-cycle, form $H$ from $G$ by deleting all vertices outside $S$; otherwise let $H=G$. Assign a charge of $5 d(v)-14$ units to each vertex $v$ of $H$ and of $2 r(f)-14$ units to each face $f$ of $H$. By Lemma 1(ii), the total charge assigned is -28 . We now redistribute the charge so that its sum is provably greater than -28 , and this contradiction will prove the theorem. Note that the charge on each face is non-negative, by the supposition that $r(g) \geqslant g \geqslant 7$; and vertices of degree $2,3,4,5, \ldots$ start with charge $-4,1,6,11, \ldots$.

Our rules for redistributing the charge are as follows:

(R1) Each distinguished 2-vertex receives 2 from each adjacent vertex.

(R2) Each distinguished 3(1)-vertex receives $\frac{1}{2}$ from each adjacent vertex that is not a distinguished 2-vertex or a distinguished 3(1)-vertex.

(R3) For each pair $b_{1}, b_{2}$ of adjacent $3(1)$-vertices, $b_{1}$ and $b_{2}$ each receive $\frac{1}{2}$ from each $>7$-face incident to edge $b_{1} b_{2}$.

It is easy to see that the charge on each face $f$ is still non-negative: by Lemma 4(iv) the boundary of $f$ contains at most $\frac{1}{3} r(f)$ pairs of adjacent 3(1)-vertices, and so $f$ gives up at most $\left\lfloor\frac{1}{3} r(f\rfloor \leqslant 2 r(f)-14\right.$ if $r(f)>7$.

We now prove that each distinguished vertex $v$ has non-negative charge. If $d(v)=2$, then $v$ started with -4 and gained 4, so now has 0 . If $d(v)=3$ then $v$ is a $3(b)$-vertex $(b \in\{0,1\})$ by Lemma 4(ii). If $b=0$ then $v$ started with 1 and gave $\frac{1}{2}$ to at most two 3(1)-vertices by Lemma 4(iii). If $b=1$ let $v$ have neighbours $v_{1}, v_{2}, v_{3}$ where $d\left(v_{1}\right)=2$. If $v_{2}$, say, is a distinguished 3(1)-vertex then, by Lemma 4(iv), $v$ received $\frac{1}{2}$ from $v_{3}$ and $\frac{1}{2}$ from the $>7$-face incident with edge $v v_{2}$ whose existence was proved in Lemma 5(ii); otherwise, $v$ received $\frac{1}{2}$ from each of $v_{2}, v_{3}$. In each case $v$ started with 1 , received at least 1 and gave at most 2 to $v_{1}$.

If $d(v)=4$ then $v$ started with 6 and, by Lemma 4(ii), gave up at most 4 to two 2 -vertices plus 1 to two 3 -vertices. If $d(v) \geqslant 5$, then $v$ gave up at most $2 d(v) \leqslant$ $5 d(v)-15$.

Now we already have a contradiction if $H=G$, when all vertices are distinguished, since in this case the sum of all charges is non-negative. If $H \neq G$ then we must also consider the vertices on $S$. Each such vertex $v$ has given at most $2(d(v)-2)$ to distinguished vertices and so now has at least $5 d(v)-14-2(d(v)-2)=3 d(v)-10$. 
This is -4 if $d(v)=2,-1$ if $d(v)=3$ and otherwise is positive. Since $G$ is 2-connected, $d(v)>2$ for at least two $v \in S$, and since $|S| \leqslant 8$ the sum of all the charges, which should be -28 , is at least $6 \times(-4)+2 \times(-1)=-26$. This contradiction completes the proof of Theorem 2 .

\title{
References
}

1. M. O. Albertson and D. Berman, 'Every planar graph has an acyclic 7-coloring', Israel J. Math. 28 (1977) 169-177.

2. I. Algor and N. Alon, 'The star arboricity of graphs', Discrete Math. 75 (1989) 11-22.

3. O. V. Borodin, 'A proof of B. Grünbaum's conjecture on acyclic 5-colourability of planar graphs', Dokl. Akad. Nauk SSSR 231 (1976) 18-20 (Russian).

4. O. V. Borodin, 'On acyclic coloring of planar graphs', Discrete Math. 25 (1979) 211-236.

5. B. GRÜNBAUM, 'Acyclic colorings of planar graphs', Israel J. Math. 14 (1973) 390-408.

6. T. JENSEN and B. ToFT, Graph coloring problems (John Wiley, New York, 1995) 38-39.

7. A. V. Kostochka, 'Acyclic 6-coloring of planar graphs', Metody Diskret. Anal. 28 (1976) 40-56 (Russian).

8. A. V. Kostochka and L. S. Melnikov, 'Note to the paper of Grünbaum on acyclic colorings', Discrete Math. 14 (1976) 403-406.

9. J. Mitchem, 'Every planar graph has an acyclic 8-coloring', Duke Math. J. 14 (1974) 177-181.

10. A. Raspaud and E. Sopena, 'Good and semi-strong colorings of oriented planar graphs', Inform. Process. Lett. 51 (1994) 171-174.

\author{
O. V. Borodin \\ Institute of Mathematics \\ Siberian Branch \\ Russian Academy of Sciences \\ Novosibirsk 630090 \\ Russia \\ D. R. Woodall \\ Department of Mathematics \\ University of Nottingham \\ Nottingham NG7 2RD
}

\author{
A. V. Kostochka \\ Novosibirsk State University \\ Novosibirsk 630090 \\ Russia
}

